\title{
PD-L1 expression levels on tumor cells affect their immunosuppressive activity
}

\author{
YANG ZHENG ${ }^{1-3^{*}}$, YOU-CHEN FANG ${ }^{1,4^{*}}$ and JING LI \\ ${ }^{1}$ Chinese Academy of Sciences Key Laboratory of Receptor Research, National Center for Drug Screening, \\ Shanghai Institute of Materia Medica, Chinese Academy of Sciences, Shanghai 201203; \\ ${ }^{2}$ School of Life Science and Technology, ShanghaiTech University, Shanghai 201210; \\ ${ }^{3}$ School of Pharmacy, University of Chinese Academy of Sciences, Beijing 100049; ${ }^{4}$ School of \\ Life Sciences, Shanghai University, Shanghai 200444, P.R. China
}

Received August 24, 2018; Accepted August 6, 2019

DOI: $10.3892 / \mathrm{ol} .2019 .10903$

\begin{abstract}
Programmed cell death 1 (PD-1) is an immunocheckpoint receptor which is primarily expressed on $\mathrm{T}$ cells, monocytes, natural killer cells and macrophages. Programmed death-ligand 1 (PD-L1) is the primary ligand of PD-1 and is constitutively expressed on antigen presenting cells, mesenchymal stem cells and bone marrow-derived mast cells. In addition, PD-L1 is also expressed on a wide range of tumor cells, including lung cancer, breast cancer and melanoma. PD-1 and PD-L1 are important members of the immunoglobulin super-family and participate in immune regulation. In the present study, the immune-suppressive effects of a number of tumor cell lines were determined. The breast tumor cell lines MCF-7 and MDA-MB-231 displayed the largest inhibitory effects on T-cell activation and cytokine secretion in a co-culture system. The HepG2, A549 and A375 cells displayed limited inhibitory effects. MCF-7 and MDA-MB-231 cells expressed the highest level of PD-L1 among the cells used, which may explain their higher immuno-suppressive effects. Compound A0-L, a small molecule inhibitor of the PD-1/PD-L1 interaction, restored $\mathrm{T}$ cell functions. Additionally, it was demonstrated that the tumor cells with higher levels of PD-L1 expression suppressed signaling pathways involved in T-cell activation, such as the T-cell receptor- zeta chain of $\mathrm{T}$ cell receptor associated protein kinase ZAP70-RAS-GTPase-extracellular-signal-regulated kinases and CD28-PI3K-Akt serine/threonine kinases pathways. These findings suggest that tumor cells with higher expression
\end{abstract}

Correspondence to: Dr Jing Li, Chinese Academy of Sciences Key Laboratory of Receptor Research, National Center for Drug Screening, Shanghai Institute of Materia Medica, Chinese Academy of Sciences, 189 Guo Shou Jing Road, Zhangjiang High-Tech Park, Pudong, Shanghai 201203, P.R. China

E-mail: lijing@simm.ac.cn

*Contributed equally

Key words: programmed cell death 1, programmed death-ligand 1, co-culture, immunosuppression, signaling pathway levels of PD-L1 may exhibit higher immuno-suppressive activity, and that drugs targeting the PD-1/PD-L1 interaction may have improved therapeutic effects on tumors expressing higher levels of PD-L1.

\section{Introduction}

Tumors utilize a variety of mechanisms to impair the functionality of tumor-specific immune cells, T cells, macrophages and other cells associated with the immune response $(1,2)$. These mechanisms include the expression of ligands which bind to inhibitory receptors expressed on $\mathrm{T}$ cells and suppressing the function of $\mathrm{T}$ cell stimulatory receptors, such as T-cell receptor (TCR)/CD3 and CD28 $(3,4)$. In general, $\mathrm{T}$ cells are activated by the interaction of the TCR/CD3 complex with an antigen and co-activation of CD28 (5). Co-stimulation of the TCR with CD28 and an antigen promotes the initial phosphorylation events of signal transduction from the TCR and enhances immune support functions (6). In addition to the foremost activation pathways, a number of immune checkpoints have been discovered to regulate the immune system. These pathways are crucial for self-tolerance and innate immunity and prevent the immune system from attacking cells indiscriminately (2). Immune checkpoints consist of stimulatory checkpoint molecules and inhibitory checkpoint molecules $(7,8)$. Inhibitory checkpoint molecules have been considered important targets for cancer immunotherapy (9). Currently, several checkpoint inhibitors which block cytotoxic T-lymphocyte associated protein 4 (CTLA4), programmed cell death-1 (PD-1) and programmed death ligand-1 (PD-L1) have been approved for clinical use (10).

The immune system regulates tumor biology, and, depending on the tumor, can either support or inhibit tumor development, growth, invasion and metastasis $(11,12)$. Certain tumors may evade immune detection through recruitment of immunosuppressive leukocytes, which create a microenvironment that blocks the antitumor immune response. Several mechanisms, including defects in antigen-presenting cells, negative immune regulation by suppressive cells and defective antitumor $\mathrm{T}$ cells have been hypothesized and demonstrated to explain evasion or tolerance of the immune response in 
different types of cancer (11). Jurkat cells are an immortalized line of human $\mathrm{T}$ lymphocyte cells that have been used to study acute T-cell leukemia and T-cell signaling (13). Jurkat cells have been used in a diverse array of molecular investigations, some of which underpin our current understanding of multiple signaling pathways (13). Evidence suggests that $\mathrm{CD} 3 / \mathrm{CD} 28$-costimulated Jurkat $\mathrm{T}$ cells and co-engagement of TCR/CD3 and CD28 results in interleukin (IL)-2 production and activation of extracellular signal regulated kinase (ERK)/c-Jun N-terminal kinase and NF- $\kappa B$ inhibitor $\beta$ kinase, which is frequently used as a functional readout of activation of Jurkat cells (14).

As an immunosuppressive molecule receptor, PD-1 can inhibit the activation of $\mathrm{T}$ lymphocytes and play an important role in immune escape. PD-1 belongs to the CD28/CTLA-4 family of molecules, and it negatively regulates PD-1 signaling. When two PD-L1 or PD-L2 ligands are concomitantly bound to PD-1, a protein tyrosine phosphatase, tyrosine-protein phosphatase non-receptor type 11 (SHP-2) is recruited intracellularly $(15,16)$. PD-L1 also termed $\mathrm{B} 7 \mathrm{H} 1$ or CD274, is primarily expressed by tumor cells and tumor-infiltrating immune cells (17), whereas PD-L2, also known as B7-DC or CD273, is expressed mainly by dendritic cells and macrophages (18). In addition to, PD-L1, but not PD-L2, undergoes a conformational change upon binding, which delays its interaction and thus activation $(19,20)$. Following PD-L1 biding to its receptor, SHP-2, dephosphorylates downstream effector molecules such as Syk and PI3K in B cells, and tyrosine-protein kinase ZAP70 (ZAP70) and CD3 in $\mathrm{T}$ cells $(21,22)$. PD-L1 is expressed in a variety of tumors $(17,23)$. PD-1/PD-L1 interaction activates a signal which inhibits TCR-mediated T-cell activation and proliferation, suppresses secretion of cytokines, such as interferon- $\gamma($ IFN- $\gamma)$ and interleukin-2, and promotes cytotoxic T-cell apoptosis and regulatory T-cell differentiation $(24,25)$. A number of pathways involved in T-cell activation, including major histocompatibility complex (MHC)-TCR-ZAP70-RAS-GTPase (RAS)-ERK and CD80-CD28-PI3K protein kinase B (AKT) pathways, have been reported to be regulated by PD-1/PD-L1 interaction.

Although several PD-1/PD-L1 inhibitors have been approved for cancer therapy, the effectiveness of these inhibitors appears to be tumor specific (26). Therefore, the aim of the present study was to determine whether the expression levels of PD-L1 on tumor cells affected its immuno-suppressive activity and thus, the therapeutic effects of PD-1/PD-L1 inhibitors. The immuno-suppressive effects of a number of tumor cell lines were assessed. It was demonstrated that MCF-7 and MDA-MB-231 cells expressed the highest levels of PD-L1 and also displayed the highest degree of immune suppression. Additionally, the tumor cells with increased levels of PD-L1 expression exhibited suppression of the pathways involved in T-cell activation, including TCR-ZAP70-RAS-ERK and CD28-PI3K-AKT pathways.

\section{Materials and methods}

Plasmid and cell lines. A PGL3-nuclear factor of activated T-cells (NFAT)-TA-Luciferase plasmid containing the full-length luciferase gene under the control of an
NFAT-driven promoter, was used in the present study. NFAT is a nuclear factor of activated $T$ cells that synergizes with activator protein 1 transcription factors at composite sites that are located in the promoters and enhancers of a number of cytokine genes. This indicates that the NFAT promoter an important factor in the immune response (27). The PGL3-NFAT-TA-Luciferase plasmid was generously provided by Dr Jia Li (Shanghai Institute of Materia Medica Chinese Academy of Science). The human embryonic kidney cell line 293, human breast cancer cell line MCF-7, human melanoma cell line A375, human cervical cancer cell line HeLa and human liver cancer cell line HepG2 were obtained from the American Type Culture Collection (ATCC) and maintained in DMEM, supplemented with $10 \%$ FBS. The human breast cancer cell line MDA-MB-231, was obtained from ATCC and maintained in Leibovitz's L-15 medium, supplemented with $10 \%$ FBS. The human lung cancer cell line A549, was obtained from ATCC and maintained in DMEM, supplemented with $10 \%$ FBS, $1 \%$ GlutaMAX ${ }^{\mathrm{TM}}$. The human T lymphocyte cell line Jurkat, was obtained from ATCC and maintained in RPMI-1640 medium, supplemented with $10 \%$ FBS, $1 \%$ GlutaMAX ${ }^{\mathrm{TM}}$ and $0.1 \%$ 2-mercaptoethanol. Human peripheral blood mononuclear cells (PBMCs) were isolated by density gradient centrifugation using a Ficoll-Paque solution (GE Healthcare) from heparinized peripheral blood samples.

Reagents. DMEM, RPMI-1640 medium, Leibovitz 15 medium, FBS, GlutaMAX ${ }^{\mathrm{TM}}$ and 2-mercaptoethanol were purchased from Gibco; Thermo Fisher Scientific, Inc. Penicillin-streptomycin was purchased from Sigma-Aldrich (Merck KGaA). FuGENE ${ }^{\circledR}$ HD Transfection Reagent was purchased from Promega Corporation and TRIzol ${ }^{\circledR}$ reagent was purchased from Invitrogen (Thermo Fisher Scientific, Inc). Anti-CD3 and anti-CD28 were purchased from BD Biosciences. Allophycocyanin-conjugated anti-human-CD274/PD-L1 antibody were purchased from eBioscience (Thermo Fisher Scientific, Inc.). Rabbit anti-Erk1/2, rabbit anti-phospho-Erk1/2, rabbit anti-AKT, rabbit anti-GAPDH and horseradish peroxidase-conjugated Goat anti-rabbit immunoglobulin $\mathrm{G}$ were purchased from Cell Signaling Technology, Inc. Human IFN- $\gamma$ ELISA kits and human IL-2 ELISA kits were purchased from Cisbio (PerkinElmer, Inc.). A0-L (a PD-1 inhibitor; patent no. WO 2015/034820 A1; molecular weight, 475.58) was synthesized by Dr Wei Lv of East China Normal University. The company name and catalog number for ELISA kits and all antibodies are listed in Table SI.

Preparation of conditioned medium. A total of $2 \times 10^{5} 293$, MCF-7, A375, A549, HeLa or HepG2 cells were cultured per well in 6-well cell culture plates with DMEM, supplemented with $10 \% \mathrm{FBS}$ at $37^{\circ} \mathrm{C}$ for $24 \mathrm{~h}$. In the A549 cells, $1 \%$ GlutaMAX $^{\mathrm{TM}}$ was added to the culture medium. MDA-MB-231 $\left(2 \times 10^{5}\right.$ cells/well) were cultured in 6 -well cell culture plates with Leibovitz's L-15 Medium, supplemented with 10\% FBS and $1 \%$ GlutaMAX $^{\mathrm{TM}}$ at $37^{\circ} \mathrm{C}$ for 24 hours. The culture media was collected and centrifuged at $12,000 \mathrm{x}$ g for $10 \mathrm{~min}$ using a pre-chilled centrifuge set to $4^{\circ} \mathrm{C}$. The supernatant was collected and termed 'conditioned medium'. 
Table I. Forward and reverse primers used for all RT-qPCR analyses.

\begin{tabular}{lll}
\hline Gene & \multicolumn{1}{c}{ Forward } & Reverse \\
\hline$G A P D H$ & AGCCGCATCTTCTTTTGCGT & TGACGAACATGGGGGCATCA \\
$P D L 1$ & GCTGCACTAATTGTCTATTGGGA & AATTCGCTTGTAGTCGGCACC \\
$I D O 1$ & GCGCTGTTGGAAATAGCTTC & ATGTCCTCCACCAGCAGTC \\
$P D L 2$ & CAGCAATGTGACCCTGGAAT & GGACTTGAGGTATGTGGAACG \\
$T I M 3$ & GGAATACAGAGCGGAGGTCG & AGGGACACATCTCCTTTGCG \\
$L A G 3$ & ACCCCATCCCAGAGGAGTTT & GTCGCCACTGTCTTCTCCAA \\
$C T L A 4$ & CCGTGCCCAGATTCTGACTT & ACATTCTGGCTCTGTTGGGG \\
$C D 86$ & TCTGTTCAGGTGTTATCCACG & GGGCGTACACTTTCCCTTCT \\
$C D 28$ & ATTCGGACAGTTGGACCCTG & CCAAGGAATGTGGTCTGGGG \\
$I C O S$ & ACACCTTTGTCCAAGTCCCC & AGCAGTGCTGCTTCTCTTACC \\
$H V E M$ & TTGAACACTGAACGCGAGGA & AAAACTGGCCAACGTGCTTC \\
$C D L A$ & GTCTTGAGGCTGGTGCTGTA & TGGTCTGGTGCTGACATTCC \\
$C D 70$ & GACCCTCCAAGGACGAAGTG & TTCTCAGGCAGCAGAACAGG \\
\hline
\end{tabular}

Co-culture of tumor cells with Jurkat cells. Jurkat cells were transfected with $3.3 \mu \mathrm{g}$ PGL3-NFAT-TA-Luciferase using FuGENE ${ }^{\circledR}$ HD Transfection Reagent. After 16 h, 2x10 $\mathrm{MCF}-7$, MDA-MB-231, A549, A375, HeLa or HepG2 cells were seeded into wells with their respective growth medium, and $2 \times 10^{4}$ Jurkat cells transfected with PGL3-NFAT-TA-Luciferase were added to the wells. The conditioned media was collected from the cultures after $24 \mathrm{~h}$. The conditioned media was added to 2x10 4 Jurkat cells transfected with PGL3-NFAT-TA-Luciferase. After $30 \mathrm{~min}$, anti-CD3 $(1 \mu \mathrm{g} / \mathrm{ml})$ and anti-CD28 $(1 \mu \mathrm{g} / \mathrm{ml})$ were added to the culture systems: Tumor cells; Jurkat cells co-cultured with tumor cells in normal media; Jurkat cells alone in conditioned media. After 24 h of co-culture, luciferase activities were measured using the Luciferase system (Promega Corporation) and EnVision multiplate reader (PerkinElmer, Inc.) according to the manufacturer's protocol.

Isolation of human PBMCs. PBMCs derived from healthy volunteers were provided by the Shanghai Blood Center. PBMCs were isolated using a Ficoll-Paque gradient. To separate PBMCs, $20 \mathrm{ml}$ Ficoll was placed in a $50 \mathrm{ml}$ conical centrifuge tube and an equal volume of whole blood diluted 1:1 with PBS was layered on top. The 50-ml tubes were centrifuged at $2,000 \mathrm{x} \mathrm{g}$ for $30 \mathrm{~min}$ at room temperature with a low acceleration speed. The PBMCs at the interface between the Ficoll and the plasma were gently collected by aspiration using a Pasteur pipette and placed in a $15 \mathrm{ml}$ conical tube. Subsequently, the PBMCs were washed twice with $10 \mathrm{ml}$ PBS and centrifuged at $500 \mathrm{x} \mathrm{g}$ for $5 \mathrm{~min}$ at $4^{\circ} \mathrm{C}(28)$. PBMCs were cultured for $6 \mathrm{~h}$ in T25 flasks in complete RPMI-1640 media (fresh RPMI-1640 medium supplemented with 10\% FBS, $2 \mathrm{mM}$ L-glutamine, $100 \mathrm{IU} / \mathrm{ml}$ penicillin and $100 \mu \mathrm{g} / \mathrm{ml}$ streptomycin and $0.1 \% 2$-mercaptoethanol) at $37^{\circ} \mathrm{C}$ in a humidified atmosphere containing $5 \% \mathrm{CO}_{2}$. The use of human PBMCs was specifically approved by The Medical Ethics Committee of Shanghai Blood Center, (Shanghai, China). Prior to donating blood, the volunteers were informed and provided written informed consent for the scientific research use of blood samples.

Co-culture of PBMCs with or without tumor cells. A total of 2x105 MCF-7, MDA-MB-231, A549, A375, HeLa, or HepG2 cells were seeded per a well in their respective growth medium for $30 \mathrm{~min}$ and then $2 \times 10^{4} \mathrm{PBMCs}$ were added to each well. Tumor cell conditioned media was collected from the cultures after $24 \mathrm{~h}$. PBMCs were exposed to tumor cell conditioned media. After $30 \mathrm{~min}$, anti-CD3 $(1 \mu \mathrm{g} / \mathrm{ml})$ and anti-CD28 $(1 \mu \mathrm{g} / \mathrm{ml})$ were added to the tumor cells/PBMCs or tumor cell conditioned media/PBMCs co-culture system. After $48 \mathrm{~h}$ of co-culture, cell culture supernatants were collected and analyzed for IL- 2 and IFN- $\gamma$ using the HTRF kit (Cisbio; PerkinElmer, Inc.) according to the manufacturer's protocol.

Reverse transcription-quantitative PCR. Total RNA was extracted using TRIzol ${ }^{\circledR}$ reagent according to the manufacturer's protocol (Invitrogen; Thermo Fisher Scientific, Inc.). RNA $(1 \mu \mathrm{g})$ was used to synthesize cDNA using a PrimeScript RT Reagent kit (Takara Bio, Inc.) according to the manufacturer's protocol. qPCR was performed for PD-L1, PD-L2, CD80, CD86, herpesvirus entry mediator (HVEM), CD70, CD137, OX40L and GAPDH. The sequences of the primer pairs used are shown in Table I. The thermocycling conditions were: $95^{\circ} \mathrm{C}$ for $10 \mathrm{~min}$; followed by 40 cycles of $95^{\circ} \mathrm{C}$ for $30 \mathrm{sec}, 60^{\circ} \mathrm{C}$ for $30 \mathrm{sec}$ and $72^{\circ} \mathrm{C}$ for $30 \mathrm{sec}$. The fold changes of each gene were calculated using the $\Delta \Delta \mathrm{Cq}$ (quantification cycle) method, and gene expression levels were normalized to GADPH (29). 
A

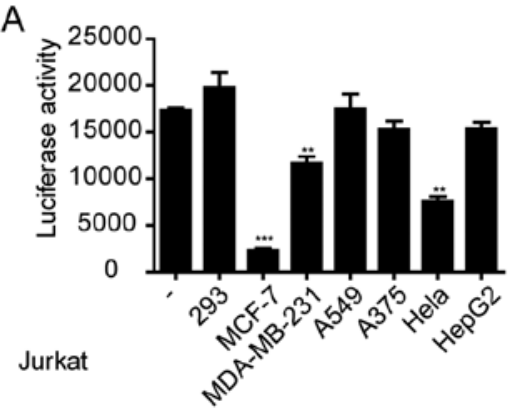

B

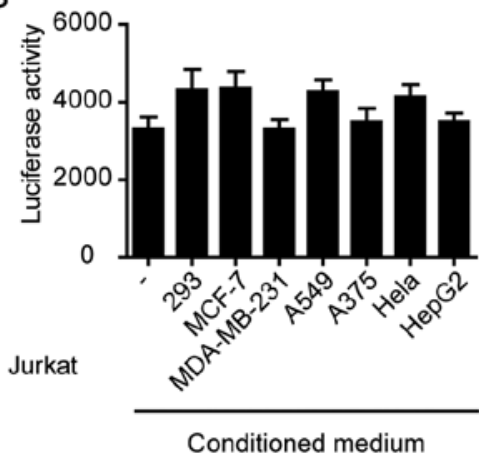

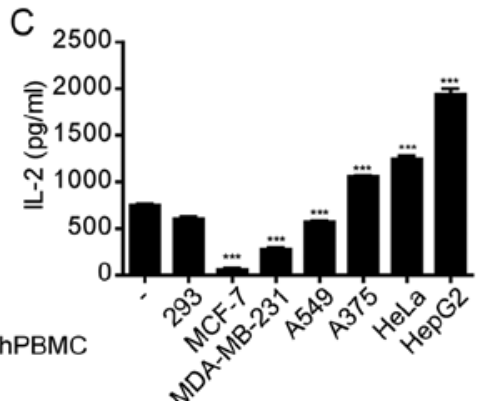

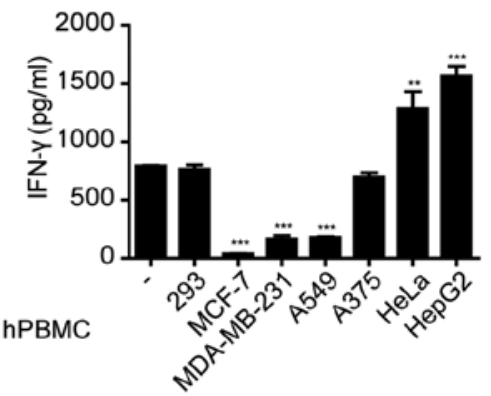

$\mathrm{D}$

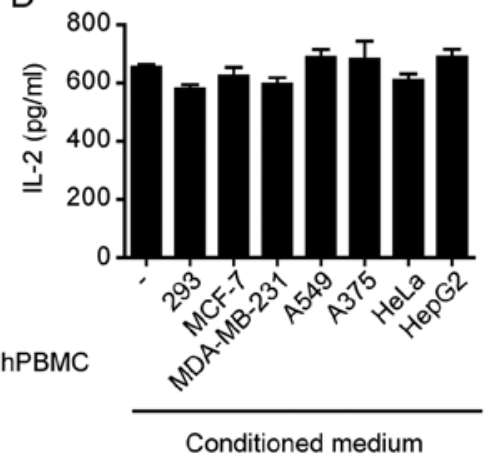

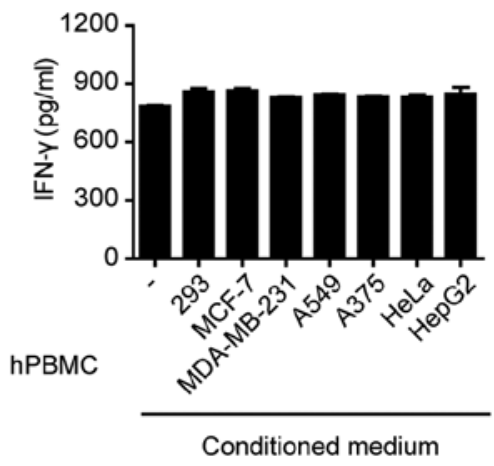

Figure 1. Immunosuppression by cancer cells. (A) Jurkat cells transfected with PGL3-NFAT-TA-Luciferase plasmid were co-cultured with various cancer cell lines and stimulated with anti-CD3 $(1 \mu \mathrm{g} / \mathrm{ml})$ and anti-CD28 $(1 \mu \mathrm{g} / \mathrm{ml})$. Luciferase activity was measured $24 \mathrm{~h}$ after stimulation. ${ }^{* *} \mathrm{P}<0.01,{ }^{* * *} \mathrm{P}<0.001 \mathrm{vs}$. control (-). (B) Jurkat cells transfected with PGL3-NFAT-TA-Luciferase plasmid were cultured in various cancer cell-conditioned media and stimulated with anti-CD3 $(1 \mu \mathrm{g} / \mathrm{ml})$ and anti-CD28 $(1 \mu \mathrm{g} / \mathrm{ml})$. Luciferase activity was measured $24 \mathrm{~h}$ after stimulation. (C) Human PBMCs were co-cultured with various cancer cell lines and then stimulated with anti-CD3 $(1 \mu \mathrm{g} / \mathrm{ml})$ and anti-CD28 $(1 \mu \mathrm{g} / \mathrm{ml}) .{ }^{* *} \mathrm{P}<0.01,{ }^{* * *} \mathrm{P}<0.001$ vs. control (-). (D) Human PBMCs cultured in various cancer cell-conditioned media were stimulated with anti-CD3 $(1 \mu \mathrm{g} / \mathrm{ml})$ and anti-CD28 $(1 \mu \mathrm{g} / \mathrm{ml})$. hPBMC, human peripheral blood mononuclear cells; IL-2, interleukin-2; IFN- $\gamma$, interferon- $\gamma$.

FACS of tumor cells. Cells were incubated with allophycocyanin-conjugated anti-human-CD274 antibody (1:100) at $4^{\circ} \mathrm{C}$ for $30 \mathrm{~min}$ in the dark for flow cytometry analysis using a Guava ${ }^{\circledR}$ easyCyte Benchtop flow cytometer and FlowJo software (FlowJo ${ }^{\mathrm{TM}}$; version 10.6.1; FlowJo LLC) was used to analyze the data.

Immunoblot analysis. MCF-7 and MDA-MB-231 cells were plated in 6 well plates at a density of $1 \times 10^{5}$ cells $/ \mathrm{ml}$. The cells were co-cultured with Jurkat cells $\left(2 \times 10^{5}\right)$ in serum-free medium, and treated with anti-CD3 $(1 \mu \mathrm{g} / \mathrm{ml})$ and anti-CD28 $(1 \mu \mathrm{g} / \mathrm{ml})$ for 5,15 and $30 \mathrm{~min}$, respectively. After the treatment, Jurkat cells were washed in PBS and lysed with RIPA lysis buffer (CoWin Biosciences). Protein concentrations were determined using a bicinchoninic acid assay kit (Thermo Fisher Scientific, Inc.). Western blot analysis was performed as previously described (30). Rabbit polyclonal antibodies against human phospho-AKT, AKT, phospho-ERK, ERK and GAPDH were used at a dilution of $1: 1,000$ at $4^{\circ} \mathrm{C}$ in the dark overnight. A horseradish peroxidase-conjugated secondary goat antibody against rabbit immunoglobulin $G$ was used at a dilution of 1:5,000 at room temperature for $1 \mathrm{~h}$. Signals were visualized using Pierce Western Blotting Substrate Plus (Thermo Fisher Scientific, Inc.) and a ChemiDocXRSp system (Bio-Rad Laboratories, Inc.).

Statistical analysis. Data were analyzed with GraphPad Prism version 5.0 (GraphPad Software, Inc.). The results were analyzed using a two-way ANOVA followed by post-hoc Bonferroni's tests or a one-way ANOVA followed by a post-hoc Newman-Keuls test. All data are presented as the mean \pm standard error of the mean. $\mathrm{P}<0.05$ was considered to indicate a statistically significant difference.

\section{Results}

Tumor cells inhibit the activation of Jurkat cells. NFATs are a family of transcription factors which serve important roles in the immune response (31). The human T lymphocyte-based Jurkat cell line expressing luciferase gene under the control of NFAT response elements can be used to study NFAT activation following various stimuli (32) To investigate the effects of the MCF-7, MDA-MB-231, A549, A375, HeLa and HepG2 cells on immune cell activation, these cells were co-cultured with Jurkat cells which were transfected with PGL3-NFAT-TA-Luciferase plasmid and stimulated with anti-CD3 and anti-CD28 antibodies. The results showed that MCF-7, MDA-MB-231 or HeLa cells significantly inhibited the anti-CD3/CD28-induced expression of luciferase in Jurkat cells (Fig. 1A), whereas the other tumor cells did not result in significant changes. However, the conditioned media collected from any of the cell lines, including MCF-7, MDA-MB-231 or HeLa cells, did not significantly affect luciferase expression in Jurkat cells (Fig. 1B). These results suggest that the direct interaction between tumor cells and Jurkat cells, rather than the factors secreted by the tumor cells, inhibited Jurkat cell activation.

Tumor cells inhibit cytokine secretion from PBMCs. Jurkat is an immortalized cell line of human T lymphocytes (13). 

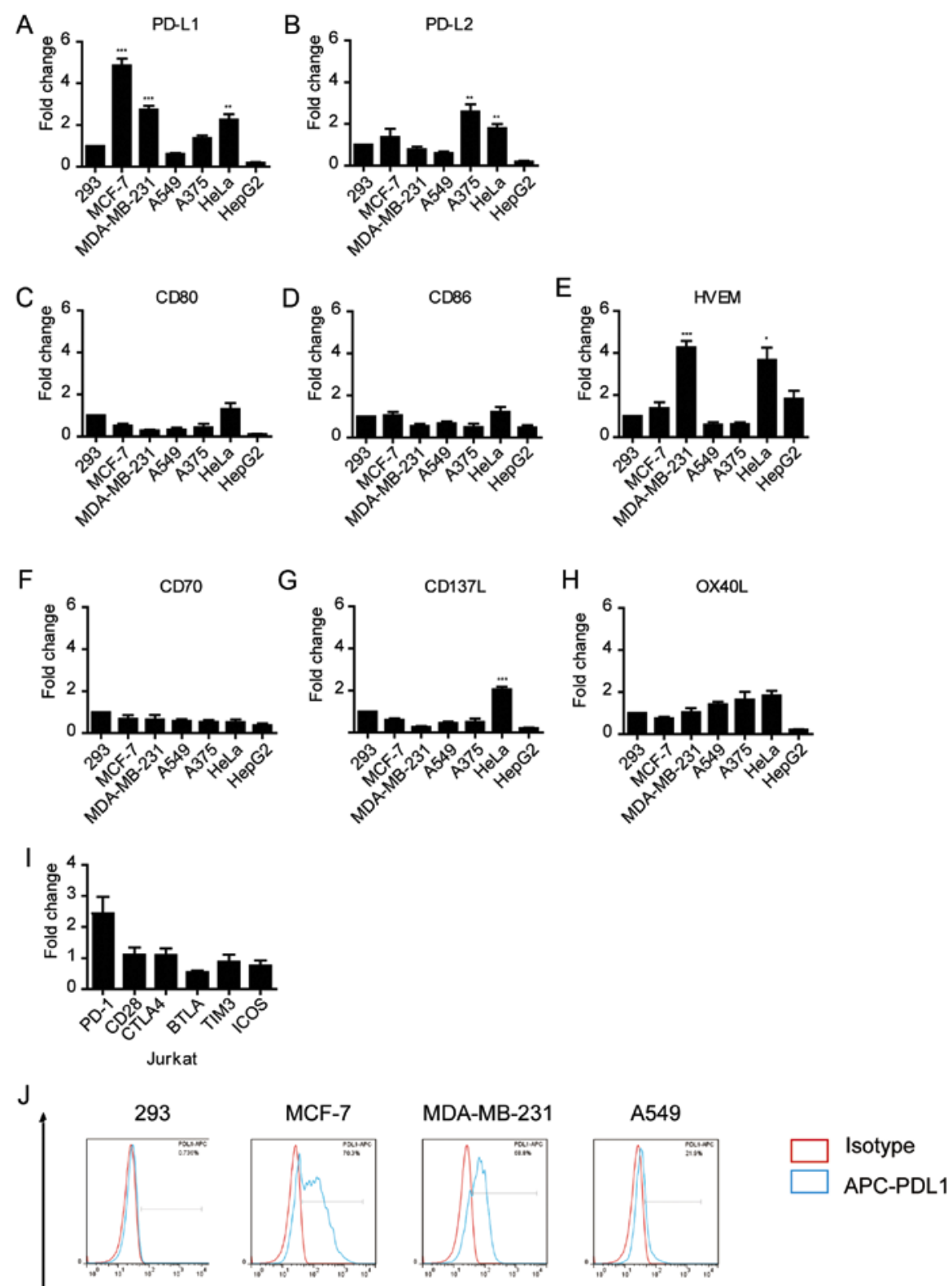

A375

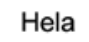

HepG2

293-PD-L1
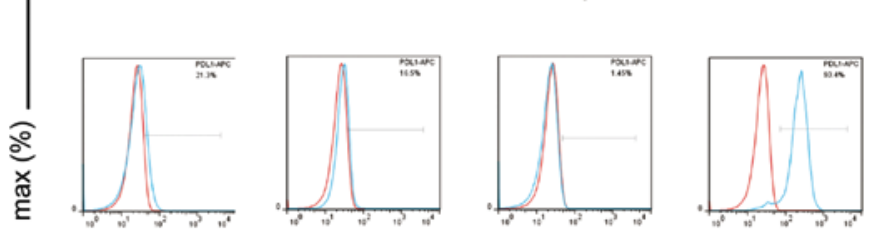

$$
\text { PD-L1 }
$$

K

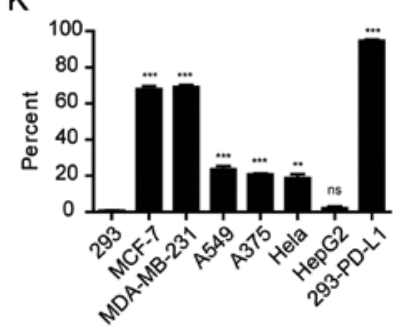

Figure 2. Expression of immune checkpoint markers in various cancer cell lines. mRNA expression levels of (A) PD-L1, (B) PD-L2, (C) CD80, (D) CD86, (E) HVEM, (F) CD70, (G) CD137 and (H) OX40L in tumor cell lines. ${ }^{*} \mathrm{P}<0.05,{ }^{* *} \mathrm{P}<0.01,{ }^{* *} \mathrm{P}<0.001$. (I) mRNA expression levels of immune checkpoint receptors in Jurkat cells. Gene expression was normalized to GAPDH in the same sample. (J) FACS analysis of PD-L1 in various cancer cell lines. APC-conjugated anti-human-PD-L1 was used as a binding antibody to cell-surface PD-L1 protein (red line, isotype control staining; blue line, PD-L1 staining). Cell count has been normalized to the peak height at the mode of the distribution, such that absolute count is presented as a percent of the total count. (K) Quantification of the FACS analysis shown in $(\mathrm{J}) .{ }^{* *} \mathrm{P}<0.01,{ }^{* * *} \mathrm{P}<0.001$ vs. 293 . APC, allophycocyanin. 
Primary PBMCs isolated from whole blood samples were used to investigate the effects of tumor cells. PBMCs were co-cultured with various tumor cell lines and stimulated with anti-CD3/CD28. The secretion of IFN- $\gamma$ and IL-2 was measured. The results showed that MCF-7, MDA-MB-231 and A549 cells significantly inhibited IFN- $\gamma$ and IL-2 secretion from PBMCs (Fig. 1C). However, the conditioned media collected from any of the cell lines, including MCF-7, MDA-MB-231 or A549 cell cultures had no effect on cytokine secretion from PBMCs (Fig. 1D). Therefore, similar to the Jurkat cells, a direct interaction between tumor cells and PBMCs resulted in the suppression of cytokine secretion.

Expression of immune checkpoint markers in various tumor cell lines. The aforementioned results suggest that different tumor cells have different effects on suppressing immune cell function. Thus, whether the expression levels of immune checkpoint proteins on tumor cells affected their immune-suppressive activity was determined. The mRNA expression levels of PD-L1 and other immune checkpoint genes, PD-L2, CD80, CD86, HVEM, CD70, CD137 and OX40L were measured in these tumor cells (Fig. 2A-H). RT-qPCR analysis demonstrated that Hela, MCF-7 and MDA-MB-231 cells expressed significantly high levels of PD-L1 compared with 293 cells $(\mathrm{P}<0.01$; Fig. 2A). PD-L1 and PD-L2 expression was significantly higher in A375 and HeLa cells $(\mathrm{P}<0.01$ and $\mathrm{P}<0.01$; Fig. $2 \mathrm{~A}$ and $\mathrm{B}$, respectively); and the expression of HVEM was significantly high in MDA-MB-231 and HeLa cells $(\mathrm{P}<0.05$; Fig. $2 \mathrm{~A}$ and $\mathrm{B})$. Notably, PD-1 was highly expressed in Jurkat cells compared with the other immune checkpoint receptors, although this was not significant (Fig. 2I). FACS analysis also confirmed that the protein expression levels of PD-L1 were considerably higher in MCF-7 and MDA-MB-231 cells compared to other tumor cells (Fig. 2J and K), consistent with the higher mRNA expression levels in these cells. Taken together, these findings suggest that the immune-suppressive activity of tumor cells may be associated with the expression levels of PD-L1 in these cells.

PD-1/PD-L1 inhibitor restores the function of lymphocytes. MCF-7 and MDA-MB-231 cells had the highest level of PD-L1 expression, and the largest inhibitory effect on T-cell activation and cytokine secretion in the co-culture system. Therefore, the two tumor cell lines were used to investigate the effect of the PD-1/PD-L1 inhibitor on the function of lymphocytes. Stimulation of Jurkat cells with anti-CD3 and anti-CD28 antibodies significantly induced the expression of luciferase, whereas MCF-7 and MDA-MB-231 cells significantly inhibited the expression of luciferase (Fig. 3A). In the present study, A0-L, a small molecular inhibitor of the PD-1/PD-L1 interaction was used. A0-L significantly restored the expression of luciferase in the tumor-Jurkat cell co-culture (Fig. 3A).

Similar results were observed in the PBMC cytokine secretion assay. Anti-CD3 and anti-CD28 co-stimulation induced IFN- $\gamma$ and IL-2 secretion from PBMC cells, which was significantly inhibited by the co-culture with MCF-7 and MDA-MB-231 cells (Fig. 3B and C). Blocking the PD-1/PD-L1 interaction between PBMCs and tumor cells with A0-L significantly increased the secretion of IFN- $\gamma$
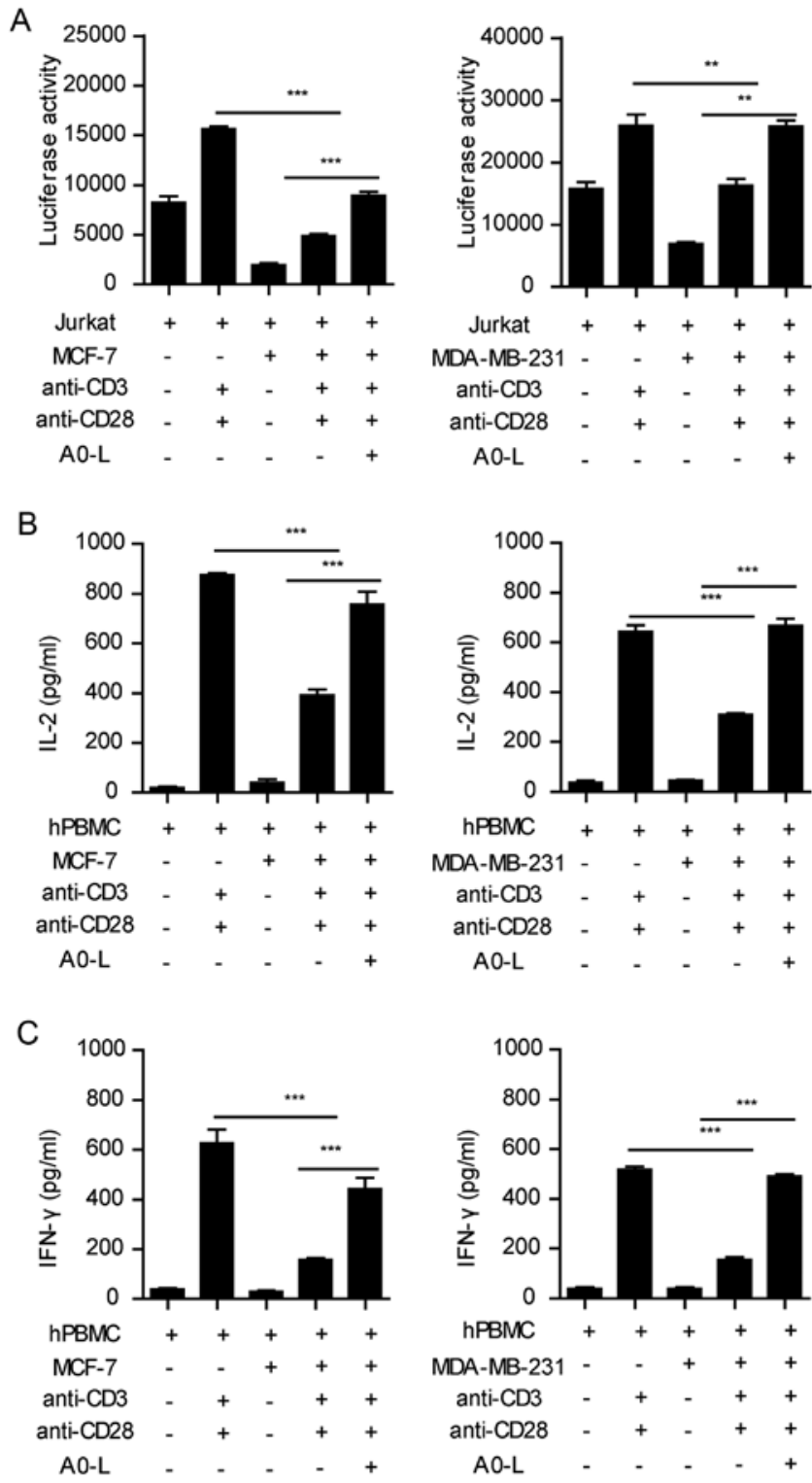

Figure 3. PD-1/PD-L1 inhibitor restores the function of lymphocytes. (A) Jurkat cells transfected with PGL3-NFAT-TA-Luciferase plasmid cocultured with various cancer cell lines were treated with A0-L $(10 \mu \mathrm{M})$ and subsequently stimulated with anti-CD3 $(1 \mu \mathrm{g} / \mathrm{ml})$ and anti-CD28 $(1 \mu \mathrm{g} / \mathrm{ml})$. Luciferase activity was measured $24 \mathrm{~h}$ later. ${ }^{* *} \mathrm{P}<0.01,{ }^{* * *} \mathrm{P}<0.001$. hPBMCs were co-cultured with MCF-7 or MDA-MB-231 in the presence of A0-L $(10 \mu \mathrm{M})$, subsequently stimulated with anti-CD3 $(1 \mu \mathrm{g} / \mathrm{ml})$ and anti-CD28 $(1 \mu \mathrm{g} / \mathrm{ml})$, and the levels of (B) IL-2 and (C) IFN- $\gamma$ were measured. ${ }_{* * * *} \mathrm{P}<0.001$. PD-1, programmed cell death 1 ; PD-L1, programmed death ligand 1 ; IL-2, interleukin-2; IFN- $\gamma$, interferon- $\gamma$; hPBMC, human peripheral blood mononuclear cells.

and IL-2 (Fig. 3B and C). Therefore, these results showed that blocking the PD-1/PD-L1 interaction effectively abrogated the inhibition of immune cell functions by tumor cells.

Tumor cells with high PD-L1 expression suppress pathways involved in T cell activation. MCF-7 or MDA-MB-231 cells were co-cultured with Jurkat cells to investigate the regulatory effects of tumor cells on AKT and ERK1/2 phosphorylation in immune cells. Jurkat cells were stimulated with anti-CD3 and anti-CD28 $(1 \mu \mathrm{g} / \mathrm{ml})$ antibodies for various durations, and AKT and ERK1/2 phosphorylation 
A

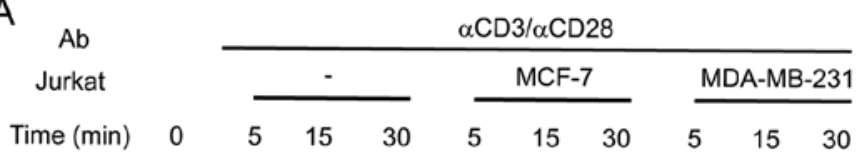

Densitometry analysis (fold change)

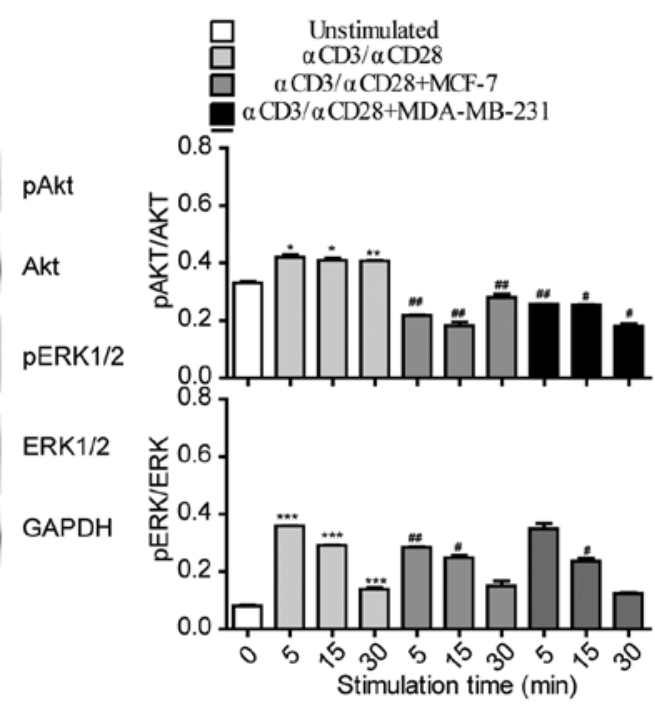

C

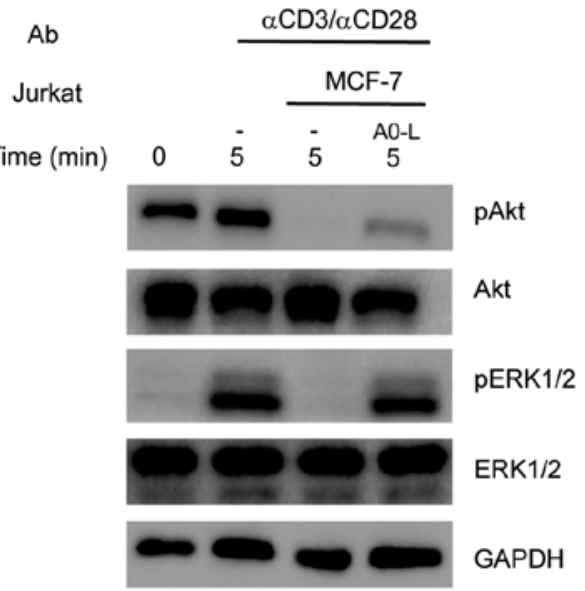

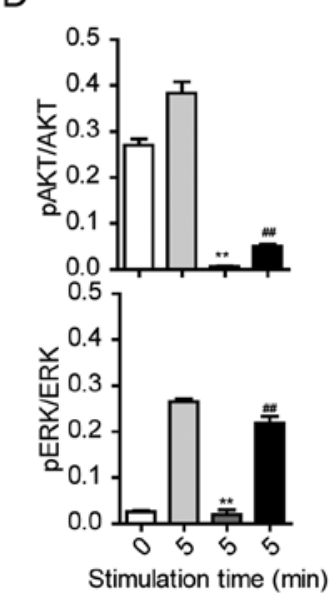

Densitometry analysis (fold change)

Unstimulated $\alpha \mathrm{CD} 3 / \alpha \mathrm{CD} 28$ $\alpha \mathrm{CD} 3 / \alpha \mathrm{CD} 28+\mathrm{MCF}-7$ $\alpha \mathrm{CD} 3 / \alpha$ CD $28+M C F-7+A 0-L$

Figure 4. Activation of AKT and ERK in Jurkat cells co-cultured with cancer cells. (A) Jurkat cells cultured alone or in the presence of MCF-7 or MDA-MB-231 cells were activated by anti-CD3 $(1 \mu \mathrm{g} / \mathrm{ml})$ and anti-CD28 $(1 \mu \mathrm{g} / \mathrm{ml})$. Jurkat cells were collected and lysates were prepared, and the amounts of the indicated proteins were determined by western blotting. (B) Densitometry analysis of the phosphorylation of AKT and ERK presented in (A). For each time point, fold changes in the amounts of the indicated proteins in activated Jurkat cells that were stimulated through anti-CD3 $(1 \mu \mathrm{g} / \mathrm{ml})$, anti-CD28 $(1 \mu \mathrm{g} / \mathrm{ml}) \mathrm{were}$ compared with cells that were not activated. ${ }^{*} \mathrm{P}<0.05,{ }^{* *} \mathrm{P}<0.01,{ }^{* * * *} \mathrm{P}<0.001$ vs. 0 min. unstimulated cells; ${ }^{\#} \mathrm{P}<0.05,{ }^{\# \#} \mathrm{P}<0.01,{ }^{\# \# \#} \mathrm{P}<0.001$ vs. Jurkat cells that were stimulated with anti-CD3 $(1 \mu \mathrm{g} / \mathrm{ml})$, anti-CD28 $(1 \mu \mathrm{g} / \mathrm{ml})$. (C) Jurkat cells cultured with MCF-7 in the absence or presence of A0-L (10 $\mu \mathrm{M})$ were activated by anti-CD3 $(1 \mu \mathrm{g} / \mathrm{ml})$ and anti-CD28 $(1 \mu \mathrm{g} / \mathrm{ml})$. Jurkat cells were collected and lysates were prepared, and the amounts of the indicated proteins were examined by western blotting. (D) Densitometry analysis of the phosphorylation of AKT and ERK presented in (C). Fold changes in the amounts of the indicated proteins in activated Jurkat cells that were suppressed by MCF-7. ${ }^{*} \mathrm{P}<0.05,{ }^{* *} \mathrm{P}<0.01,{ }^{* * *} \mathrm{P}<0.001$ vs. Jurkat cells that were stimulated through anti-CD3 ( $\left.\mu \mathrm{g} / \mathrm{ml}\right)$, anti-CD28 $(1 \mu \mathrm{g} / \mathrm{ml})$ at $5 \mathrm{~min}$. unactivated Jurkat cells that were reversed by A0-L; ${ }^{\#} \mathrm{P}<0.05,{ }^{\#} \mathrm{P}<0.01,{ }^{\# \# \#} \mathrm{P}<0.001$ vs. Jurkat cells that were stimulated with anti-CD3 $(1 \mu \mathrm{g} / \mathrm{ml})$, anti-CD28 (1 $\mu \mathrm{g} / \mathrm{ml})$ and co-cultured with MCF-7. AKT, protein kinase B; ERK, extracellular-signal regulated kinase; p, phospho; Ab, antibody.

was assessed by western blotting. Anti-CD3 and anti-CD28 were increased phosphorylation of AKT and ERK1/2 in a time-dependent manner (Fig. 4A and B). By contrast, Jurkat cells co-cultured with MCF-7 or MDA-MB-231 cells had significantly reduced phosphorylation levels of AKT and ERK1/2 following anti-CD3 and anti-CD28 stimulation (Fig. 4A and B). These results indicate that the tumor cells with high PD-L1 expression suppressed the pathways involved in T-cell activation, such as the CD28-PI3K-AKT and TCR-ZAP70-RAS-ERK pathways. Additionally, treatment with A0-L suppressed the inhibitory effects of MCF-7 cells on the CD28-PI3K-AKT and TCR-ZAP70-RAS-ERK pathways in Jurkat cells and restored phosphorylation levels of AKT and ERK1/2 (Fig. 4C and D).

\section{Discussion}

Previous findings have shown that PD-L1 is expressed on the surface of tumor cells in a number of different types of cancer and could induce immunosuppression to enable the host to evade anticancer immune responses $(33,34)$. PD-1, as an immunosuppressive factor and the receptor of PD-L1, is a critical negative regulator of cancer biology with the capacity to support cancer development, growth, invasion and metastasis (35). PD-L1 has 
also been established studied as a biomarker of a number of different types of cancer, and several studies demonstrated that PD-L1 expression may be used to predict the outcome of the disease. In the present study, the results showed that PD-L1 mRNA expression levels were upregulated in MCF-7 and MDA-MB-231 tumor cells, consistent with the high protein expression levels of PD-L1 in these cells. Additionally, MCF-7 and MDA-MB-231 cells significantly downregulated T-cell activity and cytokine secretion, which was associated with the upregulated expression of PD-L1 in these tumor cells. Blocking PD-1/PD-L1 interaction with A0-L, a PD-1/PD-L1 inhibitor, significantly restored the activation of Jurkat cells and the secretion of IFN- $\gamma$ and IL- 2 from PBMC cells, which were significantly inhibited by MCF-7 and MDA-MB-231 cells. The results suggest that PD-L1 is upregulated in specific tumor cells and may downregulate T-cell activity by binding to the PD-1 receptor on $\mathrm{T}$ cells.

T-cell activation is initiated by the binding of TCRs to their physiological ligands, which are foreign peptides bound to the MHC expressed on antigen-presenting cells (APCs) (36). Upon activation of the TCR, the Src family kinase tyrosine-protein kinase Lck (LCK) becomes activated. The activated LCK phosphorylates CD3 chains, which promote the recruitment and subsequent activation of another tyrosine kinase, ZAP-70, and recruitment of a number of other protein kinases involved in the activation of different signaling cascades, such as RAS and ERK $(37,38)$. CD28 is a co-stimulatory molecule that promotes T-cell activation (39). Upon ligand binding, CD28 recruits and activates PI3K, which in turn activates AKT by phosphorylation (40). The CD28-PI3K-AKT and TCR-ZAP70-RAS-ERK pathways are two major functional signaling pathways involved in $\mathrm{T}$ cell activation. When the TCR engages with an antigen peptide and MHC, the T cells are activated via signal transduction, and the primary signaling pathway involved is the TCR-ZAP70-RAS-ERK pathway $(41,42)$. Co-ligation of other cell surface receptors provides additional signals required to enhance T cell activation. CD28 is a costimulatory molecule that promotes $\mathrm{T}$ cell proliferation, cytokine production, cell survival and cellular metabolism. CD28-PI3K-AKT is the primary downstream signaling pathway of CD28 $(37,38)$. In the present study, it was demonstrated that tumor-T cell interaction through PD-1/PD-L1 significantly inhibited the above-mentioned pathways. Tumor cells with high PD-L1 expression inhibited TCR-dependent ERK phosphorylation and CD28-dependent AKT phosphorylation. These results suggest that PD-L1 mediates its inhibitory effects on T cell activation by regulating TCR signaling and CD28 signaling.

The PD-1/PD-L1 targeting strategy was a breakthrough in immunotherapy that restores the functions of T cells (e.g., immune cell activation and differentiation, and cytokine secretion) and promotes immune response (43-46). Keytruda and Tecentriq have been approved by the FDA, their mechanisms of action are well understood, and their clinical efficacy and pharmacodynamics data have been determined. Although several PD-1/PD-L1 inhibitors have been approved for cancer therapy, they are more effective in treating certain tumors over others (47). The results of the present study suggest that tumor cells with a higher expression level of PD-L1 may have higher immunosuppressive activity, and drugs targeting the PD-1/PD-L1 interaction may have improved therapeutic effects on tumors with higher expression levels of PD-L1.

\section{Acknowledgements}

Not applicable.

\section{Funding}

The present study was supported by grants from The National Science \& Technology Major Project: Key New Drug Creation and Manufacturing Program (Beijing, China; grant no. 2017ZX09101004-012-008); Personalized Medicines-Molecular Signature-based Drug Discovery and Development; Strategic Priority Research Program of the Chinese Academy of Sciences (Beijing, China; grant no. XDA12040212), and Shanghai Commission of Science and Technology (Shanghai, China; grant no. 16431901500).

\section{Availability of data and materials}

The datasets used and/or analyzed during the present study are available from the corresponding author on reasonable request.

\section{Authors' contribution}

YZ performed the majority of the experiments, analyzed the data and wrote the manuscript. YCF performed some of the experiments. JL supervised the study, analyzed the data and wrote the manuscript. All authors read and approved the final manuscript.

\section{Ethics approval and consent to participate}

The use of human PBMCs was specifically approved by The Medical Ethics Committee of Shanghai Blood Center, (Shanghai, China). Prior to donating blood, the volunteers were informed and provided written informed consent for the scientific research use of blood samples. Due to ethical constraints, no clinical information on the blood donors was obtained.

\section{Patient consent for publication}

Not applicable.

\section{Competing interests}

The authors declare that they have no competing interests.

\section{References}

1. Ahmadzadeh M, Johnson LA, Heemskerk B, Wunderlich JR, Dudley ME, White DE and Rosenberg SA: Tumor antigen-specific CD8 T cells infiltrating the tumor express high levels of PD-1 and are functionally impaired. Blood 114: 1537-1544, 2009.

2. Prosser ME, Brown CE, Shami AF, Forman SJ and Jensen MC: Tumor PD-L1 co-stimulates primary human CD8(+) cytotoxic $\mathrm{T}$ cells modified to express a PD1:CD28 chimeric receptor. Mol Immunol 51: 263-272, 2012.

3. June $\mathrm{CH}$, Ledbetter JA, Linsley PS and Thompson CB: Role of the CD28 receptor in T-cell activation. Immunol Today 11: 211-216, 1990. 
4. Alvarez-Vallina L and Hawkins RE: Antigen-specific targeting of CD28-mediated $\mathrm{T}$ cell co-stimulation using chimeric single-chain antibody variable fragment-CD28 receptors. Eur J Immunol 26: 2304-2309, 1996.

5. Louis-Dit-Sully C, Blumenthal B, Duchniewicz M, Beck-Garcia K, Fiala GJ, Beck-García E, Mukenhirn M, Minguet S and Schamel WW: Activation of the TCR complex by peptide-MHC and superantigens. Exs 104: 9-23, 2014.

6. Salazar-Fontana LI, Barr V, Samelson LE and Bierer BE: CD28 engagement promotes actin polymerization through the activation of the small Rho GTPase Cdc42 in human T cells. J Immunol 171: 2225-2232, 2003.

7. Passardi A, Canale M, Valgiusti M and Ulivi P: Immune checkpoints as a target for colorectal cancer treatment. Int J Mol Sci 18 : pii: E1324, 2017

8. Saad FT, Hincal E and Kaymakamzade B: Dynamics of immune checkpoints, immune system, and BCG in the treatment of superficial bladder cancer. Comput Math Methods Med 2017: $3573082,2017$.

9. Sharma P and Allison JP: Immune checkpoint targeting in cancer therapy: Toward combination strategies with curative potential. Cell 161: 205-214, 2015.

10. Pardoll DM: The blockade of immune checkpoints in cancer immunotherapy. Nat Rev Cancer 12: 252-264, 2012.

11. Beatty GL and Gladney WL: Immune Escape Mechanisms as a Guide for Cancer Immunotherapy. Clin Cancer Res 21: 687-692, 2015.

12. Janssen LME, Ramsay EE, Logsdon CD and Overwijk WW: The immune system in cancer metastasis: Friend or foe? J Immunother Cancer 5: 79, 2017.

13. Bartelt RR, Cruz-Orcutt N, Collins M and Houtman JC: Comparison of $\mathrm{T}$ cell receptor-induced proximal signaling and downstream functions in immortalized and primary $\mathrm{T}$ cells. PLoS One 4: e5430, 2009.

14. Thaker YR, Schneider H and Rudd CE: TCR and CD28 activate the transcription factor NF- $\kappa \mathrm{B}$ in T-cells via distinct adaptor signaling complexes. Immunol Lett 163: 113-119, 2015.

15. Dong H, Zhu G, Tamada K and Chen L: B7-H1, a third member of the B7 family, co-stimulates T-cell proliferation and interleukin-10 secretion. Nat Med 5: 1365-1369, 1999.

16. Latchman Y, Wood C, Chemova T, Chaudhary D, Borde M, Chernova I, Iwai Y, Long AJ, Brown JA, Nunes R, et al: PD-L2, a novel B7 homologue, is a second ligand for PD-1 and inhibits T cell activation. Nat Immunol 15: 261-268, 2001.

17. Kim HR, Ha SJ, Hong MH, Heo SJ, Koh YW, Choi EC, Kim EK, Pyo KH, Jung I, Seo D, et al: PD-L1 expression on immune cells, but not on tumor cells, is a favorable prognostic factor for head and neck cancer patients. Sci Rep 6: 36956, 2016.

18. Keir ME, Liang SC, Guleria I, Latchman YE, Qipo A, Albacker LA, Koulmanda M, Freeman GJ, Sayegh MH and Sharpe AH: Tissue expression of PD-L1 mediates peripheral T cell tolerance. J Exp Med 203: 883-895, 2006.

19. Butte MJ, Keir ME, Phamduy TB, Sharpe AH and Freeman GJ: Programmed death-1 ligand 1 interacts specifically with the B7-1 costimulatory molecule to inhibit $\mathrm{T}$ cell responses. Immunity 27 $111-122,2007$.

20. Ghiotto M, Gauthier L, Serriari N, Pastor S, Truneh A, Nunès JA and Olive D: PD-L1 and PD-L2 differ in their molecular mechanisms of interaction with PD-1. Int Immunol 22: 651-660, 2010.

21. Zheng P and Zhou Z: Human cancer immunotherapy with PD-1/PD-L1 blockade. Biomark Cancer 7 (Suppl 2): S15-S18, 2015.

22. Catakovic K, Klieser E, Neureiter D and Geisberger R: T cell exhaustion: From pathophysiological basics to tumor immunotherapy. Cell Commun Signal 15: 1, 2017.

23. Patel SP and Kurzrock R: PD-L1 expression as a predictive biomarker in cancer immunotherapy. Mol Cancer Ther 14 847-856, 2015.

24. Palaga T, Miele L, Golde TE and Osborne BA: TCR-mediated Notch signaling regulates proliferation and IFN-gamma production in peripheral T cells. J Immunol 171: 3019-3024, 2003.

25. Iwasaki M, Tanaka Y, Kobayashi H, Murata-Hirai K, Miyabe H Sugie T, Toi M and Minato N: Expression and function of PD-1 in human $\gamma \delta \mathrm{T}$ cells that recognize phosphoantigens. Eur $\mathrm{J}$ Immunol 41: 345-355, 2011.

26. Wang D, Lin J, Yang X, Long J, Bai Y, Yang X, Mao Y, Sang X, Seery S and Zhao H: Combination regimens with PD-1/PD-L1 immune checkpoint inhibitors for gastrointestinal malignancies. J Hematol Oncol 12: 42, 2019.
27. Macian F: NFAT proteins: Key regulators of T-cell development and function. Nat Rev Immunol 5: 472-484, 2005.

28. Grievink HW, Luisman T, Kluft C, Moerland M and Malone KE Comparison of three isolation techniques for human peripheral blood mononuclear cells: Cell recovery and viability, population composition, and cell functionality. Biopreserv Biobank 14: 410-415, 2016.

29. Livak KJ and Schmittgen TD: Analysis of relative gene expression data using real-time quantitative PCR and the 2(-Delta Delta C(T)) method. Methods 25: 402-408, 2001.

30. Patsoukis N, Brown J, Petkova V, Liu F, Li L and Boussiotis VA: Selective effects of PD-1 on Akt and Ras pathways regulate molecular components of the cell cycle and inhibit T cell proliferation. Sci Signal 5: ra46, 2012.

31. Vaeth $M$ and Feske S: NFAT control of immune function: New frontiers for an abiding trooper. F1000Res 7: 260, 2018

32. Ponomarev V, Doubrovin M, Lyddane C, Beresten T, Balatoni J, Bornman W, Finn R, Akhurst T, Larson S, Blasberg R, et al: Imaging TCR-dependent NFAT-mediated T-cell activation with positron emission tomography in vivo. Neoplasia 3: 480-488, 2001

33. Ding ZC, Lu XY, Yu M, Lemos H, Huang L, Chandler P, Liu K, Walters M, Krasinski A, Mack M, et al: Immunosuppressive myeloid cells induced by chemotherapy attenuate antitumor CD4+ T-Cell Responses through the PD-1-PD-L1 Axis. Cancer Res 74: 3441-3453, 2014.

34. Medina PJ and Adams VR: PD-1 Pathway Inhibitors: Immuno-oncology agents for restoring antitumor immune responses. Pharmacotherapy 36: 317-334, 2016.

35. Liu Y and Cao XT: Immunosuppressive cells in tumor immune escape and metastasis. J Mol Med 94: 509-522, 2016.

36. Dustin ML: The cellular context of T cell signaling. Immunity 30 : 482-492, 2009

37. Zhu X, Kim JL, Newcomb JR, Rose PE, Stover DR, Toledo LM, Zhao H and Morgenstern KA: Structural analysis of the lymphocyte-specific kinase Lck in complex with non-selective and Src family selective kinase inhibitors. Structure 7: 651-661, 1999.

38. Li M, Ong SS, Rajwa B, Thieu VT, Geahlen RL and Harrison ML: The SH3 domain of Lck modulates T-cell receptor-dependent activation of extracellular signal-regulated kinase through activation of Raf-1. Mol Cell Biol 28: 630-641, 2008

39. Beyersdorf N, Kerkau T and Hunig T: CD28 co-stimulation in T-cell homeostasis: A recent perspective. Immunotargets Ther 4: 111-122, 2015.

40. Parry RV, Riley JL and Ward SG: Signalling to suit function: Tailoring phosphoinositide 3 -kinase during T-cell activation. Trends Immunol 28: 161-168, 2007.

41. Butler DE, Marlein C, Walker HF, Frame FM, Mann VM, Simms MS, Davies BR, Collins AT and Maitland NJ: Inhibition of the PI3K/AKT/mTOR pathway activates autophagy and compensatory Ras/Raf/MEK/ERK signalling in prostate cancer. Oncotarget 8: 56698-56713, 2017.

42. Asati V, Mahapatra DK and Bharti SK: PI3K/Akt/mTOR and Ras/Raf/MEK/ERK signaling pathways inhibitors as anticancer agents: Structural and pharmacological perspectives. Eur J Med Chem 109: 314-341, 2016.

43. Mahoney KM, Freeman GJ and McDermott DF: The next immune-checkpoint inhibitors: PD-1/PD-L1 blockade in melanoma. Clin Ther 37: 764-782, 2015.

44. Hui E, Cheung J, Zhu J, Su X, Taylor MJ, Wallweber HA, Sasmal DK, Huang J, Kim JM, Mellman I and Vale RD: $\mathrm{T}$ cell costimulatory receptor $\mathrm{CD} 28$ is a primary target for PD-1-mediated inhibition. Science 355: 1428-1433, 2017.

45. Jacquin-Porretaz C, Nardin C, Puzenat E, Roche-Kubler B, Aubin F; Comité de suivi des effets secondaires des immunothérapies anti-cancéreuses(CSESIAC): Adverse effects of immune checkpoint inhibitors used to treat melanoma and other cancer. Presse Med 46: 808-817, 2017 (In French).

46. Park JA and Cheung NV: Erratum to 'limitations and opportunities for immune checkpoint inhibitors in pediatric malignancies' [Cancer Treat. Rev. 58C (2017) 22-33]. Cancer Treat Rev 60: 158, 2017.

47. McDermott DF and Atkins MB: PD-1 as a potential target in cancer therapy. Cancer Med 2: 662-673, 2013.

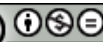

This work is licensed under a Creative Commons Attribution-NonCommercial-NoDerivatives 4.0 International (CC BY-NC-ND 4.0) License. 